Original paper

\title{
Developing and implementing a multi-modality imaging optimization study in paediatric radiology: Experience and recommendations from an IAEA coordinated research project
}

\author{
H. Delis ${ }^{a, *}$, P. Homolka ${ }^{\text {b }}$, C.L. Chapple ${ }^{c}$, P.R. Costa ${ }^{\text {d }}$, E. Attalla ${ }^{e}$, L.E. Lubis ${ }^{f}$, T.A. Sackey ${ }^{g}$, \\ F. Fahey ${ }^{\text {h, M. Lassmann }}{ }^{\mathrm{i}}$, G.L. Poli ${ }^{\mathrm{a}}$ \\ ${ }^{a}$ Dosimetry and Medical Radiation Physics Section, Division of Human Health, International Atomic Energy Agency, Vienna, Austria \\ ${ }^{\mathrm{b}}$ Center for Medical Physics and Biomedical Engineering, Medical University of Vienna, Vienna, Austria \\ ${ }^{\mathrm{c}}$ Newcastle upon Tyne Hospitals NHS Trust, Newcastle upon Tyne, United Kingdom \\ ${ }^{\mathrm{d}}$ Institute of Physics, University of São Paulo, São Paulo, Brazil \\ e Medical Physics Unit, Department of Radiotherapy, National Cancer Institute, Cairo University, Egypt \\ ${ }^{\mathrm{f}}$ Department of Physics, Faculty of Mathematics and Natural Sciences, Universitas Indonesia, Depok, Indonesia \\ ${ }^{g}$ Radiological and Medical Sciences Research Institute, Ghana Atomic Energy Commission, Accra, Ghana

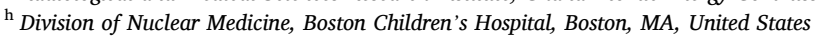 \\ ${ }^{i}$ Department of Nuclear Medicine, University Hospital Würzburg, Würzburg, Germany
}

\section{A R T I C L E I N F O}

\section{Keywords:}

Paediatric radiology

Optimization

Dosimetry

\begin{abstract}
A B S T R A C T
Optimization of imaging examinations is a key requirement of both the International and European Basic Safety Standards, and the focus of much international activity. Although methodologies are well established in principle, there continues to be a variety of practical issues both in collecting and interpreting dose and image quality data and in making successful interventions to optimize exposures.

A Coordinated Research Project, involving institutes from ten different countries, was established by the IAEA to assess the efficacy of recommended optimization methodologies in the field of paediatric radiology and to derive practical guidance on their implementation. The steps followed in this process were identification of the imaging process to be investigated (abdomen and chest x-rays, micturating cysto-urethrograms, and brain \& thorax CT scans); collection of dose and image quality data; evaluation and comparison of the data between institutes and to standards; identification and implementation of interventions for optimization; and reevaluation of dose and image quality parameters.

The project succeeded both in achieving effective interventions for optimization of specific imaging tasks in individual institutes and in identifying key issues with potential to handicap this process. The main area in which problems were encountered was in the collation of reliable dose and image quality data. The reasons for this were explored and a series of recommendations have been made, summarized into 'ten practical tips' for optimization to assist institutes, particularly those in the early stages of addressing optimization issues.
\end{abstract}

\section{Introduction}

Optimization of medical imaging procedures is a fundamental requirement of quality practices and a key part of the International Basic Safety Standards [1]. This has also been clearly acknowledged in the Bonn Call for Action [2] which identifies optimization as one of its main Actions.

Careful evaluation of practices that will lead to optimization of these can be motivated by a number of different factors, such as:

- Need for continuous quality improvement of practices

- Contribution to Diagnostic Reference Levels

- Regulatory requirements

Methodologies for the evaluation and optimization processes have been published in the past, including guidance for paediatric patients

\footnotetext{
* Corresponding author.

E-mail address: dosimetry@iaea.org (H. Delis).
} 
[3-12]. To evaluate the applicability and difficulties of these approaches for dose audits, data collection and optimization in a wide range of clinical settings the International Atomic Energy Agency in 2015 initiated a Coordinated Research Project (CRP) on "Evaluation and Optimization of Paediatric Imaging". The CRP, that includes both Diagnostic Radiology and Nuclear Medicine practices, was aiming to enhance the capabilities of the participating institutes to improve the efficiency of existing modalities for paediatric medical imaging, as well as to implement and enhance optimization techniques and methodologies for advanced paediatric medical imaging. The ultimate benefit of this CRP is to the large number of paediatric patients undergoing diagnostic procedures with ionizing radiation.

During the process of optimization, it is crucial to keep in mind that the objective of medical imaging is the diagnostic information of the image and thus numerical dose values cannot give a full view of the practices, unless adequately supported by image quality evaluation. Furthermore, dose data, as numerical values, are not an appropriate indication of better or worse practice but can, to some extent, reveal useful information regarding weaknesses and gaps in local practices that can be improved or corrected. In addition, the identification of patterns of dose quantities is a milestone for a consistent optimization process in paediatric patients, since the quantification of absorbed dose in representative organs is complex, especially in CT procedures [13-15].

Institutes from 10 IAEA Member States (Austria, Brazil, Chile, Cuba, Egypt, Germany, Ghana, Indonesia, United Kingdom and United States) were selected to be part of this coordinated CRP and contributed in different parts of the research, with 6 institutes from 5 Member States (Austria, Brazil, Egypt, Indonesia and United Kingdom) contributing data for the work presented here. The optimization studies carried out as part of the CRP all focused on paediatric imaging, involving general radiography (GR), fluoroscopy, computed tomography (CT) and nuclear medicine (NM) examinations $[16,17]$. Although participating institutes are not necessarily representing the entirety of practice in their countries, they provide the wide spectrum of different populations, practices and technologies that is necessary for this evaluation.

This paper will focus on the experience from this project in Diagnostic Radiology and can be used as an educational tool to provide practical guidance on developing an optimization study and how to carry it out successfully, with illustrations from the work that was carried out and the lessons learnt. The experience acquired by the CRP participants can be used as a practical exemplification of the potential benefits resulting from the implementation of a well-structured optimization strategy, which should include the "who" (roles and responsibilities), "when" (detailed time frame) and "how" (analytical protocols) of each step of the optimization process. The key points requiring close attention for a successful optimization practice in paediatric radiology will be outlined.

\section{Materials and methods}

The methodology for optimization is, in principle, a straightforward process, and the steps are outlined in Fig. 1 and will be discussed in more detail below. The methodology described represents an example of the application of the plan-do-check-act cycle. In different situations the process can be adapted, but the general ideas - and pitfalls - identified in the process of the coordinated research project provide a valuable guidance.

After the planning of the optimization process, the initial data collection is essentially the starting point for a detailed analysis in a focused and critical way, comparing the local situation with the recommendations that concern good practice, in order to identify possible gaps or areas for improvement. Once these are identified, suitable interventions can be agreed and put in practice, preferably in the form of updated documented procedures that provides common guidance for all staff. Then this new situation has to be again evaluated and the corresponding results compared to the initial benchmark data, to assess the impact of the intervention, as an analogue of the quality PDCA (plan-docheck-act) process.

\subsection{Identification of imaging situation for optimization}

To carry out an effective optimization study it is necessary to be specific about the scope of work, with respect both to patient cohort (e.g. paediatric patients, standard sized adults, bariatric patients) and also the particular examination [18].

During the CRP, this stage was carried out as a specific exercise, along with a detailed plan of methodology. Types of paediatric examination to be considered were carefully selected based on potential

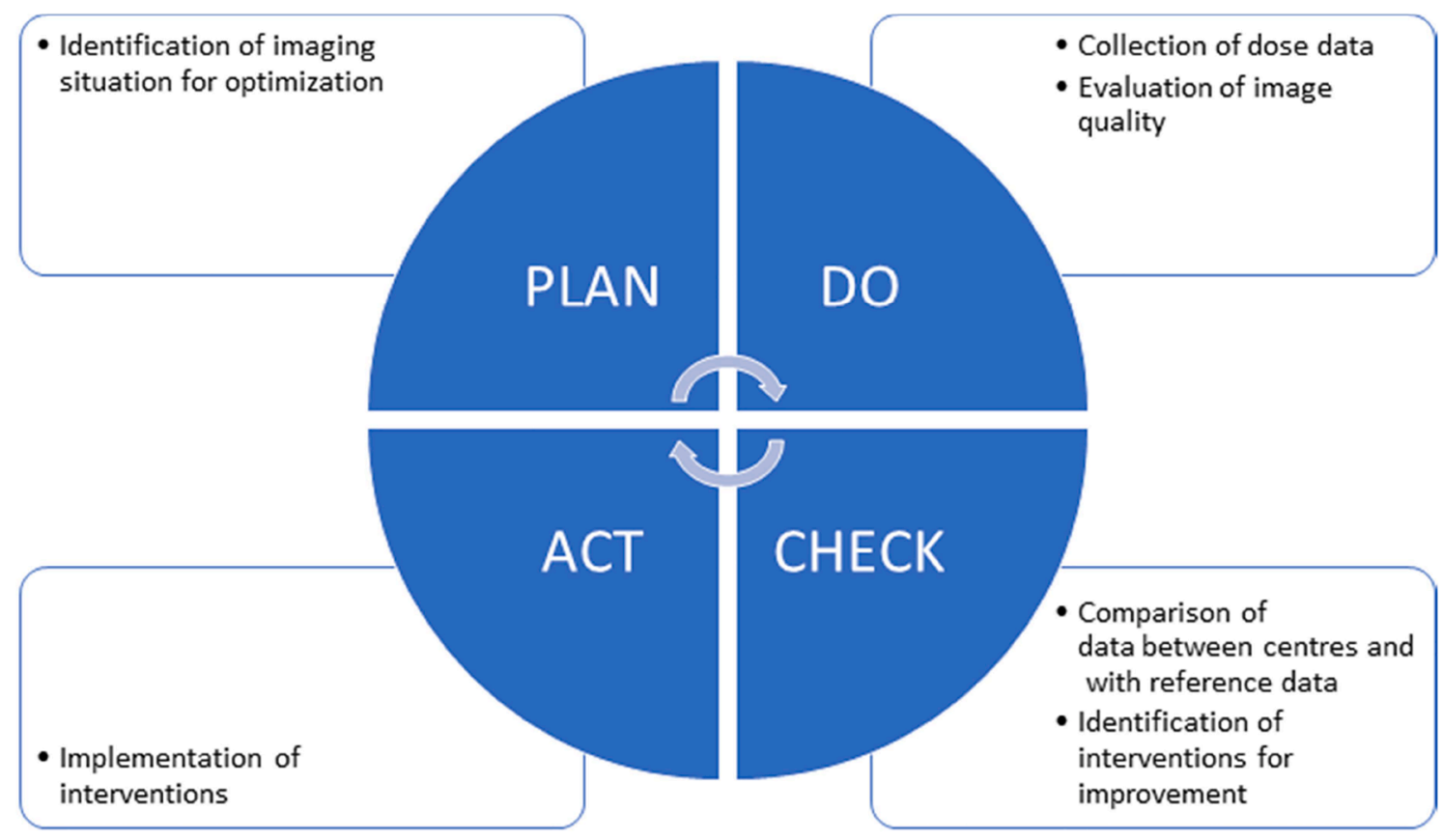

Fig. 1. Optimization process. 
frequency and/or expected dose levels, as well as availability of a standard protocol in each participating institute. For general radiography, abdomen (AP) and chest examinations (AP-PA) were selected. In $\mathrm{CT}$, non-contrast brain and thorax examinations were chosen, and micturating cysto-urethrogram (MCU) in fluoroscopy. Collected patient data were defined in age ranges to enable single analysis and averaging within each age range. Referring to existing recommendations $[9,19]$ the determined age ranges were $0-1$ years, $1-5$ years, $5-10$ years, and $10-15$ years across modalities.

\subsection{Collection of dose data}

A number of different quantities are available for assessing radiation exposure from imaging procedures. For general radiography and fluoroscopy, air kerma-area-product (KAP) is generally used. Alternatively, the incident air kerma can be used in projection radiography, although it misses information about the field size used. For CT examinations the appropriate quantities are Computed Tomography Dose Index (CTDI) and dose length product (DLP). KAP and DLP were selected as dosimetric quantities in this CRP.

Within the CRP, data sheets [9] were adapted for the project. The use of standardized sheets helped to ensure uniformity of the data collection. It was also required that quality control tests were carried out on each equipment and the QC test methodology and the units used were included in the data collection sheets. As it is of fundamental importance that dose meters are adequately calibrated, or displayed values verified within a known tolerance, relevant forms and procedures for the calibration of dose monitoring equipment [20] were distributed amongst participants and data collection sheets had this highlighted as an initial requirement, prior to the dose data collection.

The dose parameters used were collated in a number of different ways across participants, reflecting the spread of methodologies generally available. Increasingly prevalent was the use of electronically recorded parameters, determined by the imaging equipment itself, transferred to the hospital's patient or imaging data management system, and automatically downloaded into a spreadsheet or using commercial software. At the other end of the spectrum, the dose quantities were calculated manually from more easily acquired data relating to individual patient exposures following the procedures described in relevant references $[9,20,21]$. In between these extremes, electronic dose data were transcribed manually, or semi-automatically, either from a dose measuring device, the imaging equipment itself, or from a data management system. In all cases, independently of the adopted modality for data recording, careful measurements were used in order to validate the dose parameters according to TRS 457 [20] using instruments with a traceable calibration. This verification step was part of the initial quality control tests required by participants.

\subsection{Evaluation of image quality}

Assessment of image quality may be carried out by either qualitative or quantitative means. While quantitative measures, such as Signal to Noise Ratio or Modulation Transfer Function, allow for direct comparisons to be made, it is generally accepted that no one of these provides an adequate measure of acceptability of a diagnostic image, for which a subjective assessment of clinical diagnosticity is required. Such subjective assessment however depends greatly on both the evaluator and the clinical question, so will often not be comparable between centres.

For the purposes of the CRP, image quality for each image included for dose data collection was required to be assessed by a radiologist through assignment of an Image Quality Factor using a modified 3 grade Likert scale. The definition of this scale used was similar to the nuclear medicine optimization study [16] with:

\section{IQ3: Sufficient image quality.}

Acceptable image IQ1 was communicated to be used in cases where the image could not be used for appropriate diagnosis, and a rescan or retake should be requested. IQ2 was used in cases, where clinicians could actually diagnose the images, but expressed their concern about image quality. IQ3 was to be used if clinicians were fully satisfied with the image quality. IQ3, with respect to the ALARA principle, indicated images with an appropriate noise level to allow diagnostic confidence.

\subsection{Comparison of data between centres and with reference data}

Dose and image quality data need to be assessed as to whether they are at an appropriate level, in order to determine any requirement for optimization. Diagnostic Reference Levels (DRLs) are a useful tool for assessing dose, and local, national or international values may be used for this purpose where available. If data are collected from multiple rooms in the same institute, or from multiple institutes, these may be directly compared with one another to assess where each lies within the range of practice.

Within the CRP, data from the different participants were sent to the central coordinator, who performed initial checks of the format, the consistency and plausibility of the data, collated the data and performed statistical analysis. Dose data were compared for each examination type and age group and presented on statistical graphs to give an indication of where substantial differences in dose occurred, and how these compared with internationally available paediatric DRLs. This was most evident in data received from Centers $\mathrm{A}$ and $\mathrm{C}$ where some dose data differed approximately by one order of magnitude or more from expected values, which triggered instant checks of data quality. Dose data reported by the participants was compared to DRLs based on the European Guidelines on Diagnostic Reference Levels for Paediatric Imaging, Radiation Protection 185 ([22], Table 1), although these values are not necessarily relevant to all participating countries. However, Radiation Protection 185 provides values based on weight bands (for all modalities and examinations except head CT) or age bands (head CT) defined differently to the ones used in this project. Therefore, these values have been interpolated to fit the weight bands used in this work (Table 2) using reference data on age/weight relations from Rodd et al. [23]. To visualize systematic deviations from DRLs the ratios of the relevant patient dose indicators to the corresponding DRLs were plotted for all age groups to better illustrate their dependence on patient age within a single institution.

In addition to comparing dose information, comparisons of imaging techniques were carried out to help determine the reasons for any observed differences. Protocol data was also considered during the data collection and comparison phases. Where dose differences were not readily explicable from differences in equipment or protocol, data were further checked for accuracy and consistency, in particular regarding the adopted units, prior to another analysis.

\subsection{Identification of interventions for improvement}

In diagnostic imaging optimization, the identification of necessary interventions is one of the most important steps in the optimization cycle (Fig. 1) that must be considered with the utmost diligence. Once an area for improvement is identified, based on either dose or image quality, there is a need to consider how to achieve it. This will normally be apparent from a consideration of differences in relevant exposure parameters like $\mathrm{kVp}$, mAs or reference mAs, pulse rate in fluoroscopy, or dose descriptors like KAP or DLP. A pre-requisite to this process is to ensure an operational QA programme is in place, as this provides necessary system data relating to both dose and image quality 
Table 1

Reference levels according to Radiation Protection 185 [22]. Note: all values but head CT are provided in weight bands (head CT in age bands).

\begin{tabular}{|c|c|c|c|c|c|c|c|}
\hline \multirow[t]{2}{*}{ Modality } & \multicolumn{7}{|l|}{ Examination } \\
\hline & & Weight band & $<5 \mathrm{~kg}$ & $5-15 \mathrm{~kg}$ & $15-30 \mathrm{~kg}$ & $30-50 \mathrm{~kg}$ & $50-80 \mathrm{~kg}$ \\
\hline \multirow[t]{2}{*}{ Radiography } & Thorax AP/PA & $\mathrm{KAP}$ in $\mu \mathrm{Gy} \cdot \mathrm{m}^{2}$ & $1.5^{\circ}$ & 2.2 & 5.0 & 7.0 & 8.7 \\
\hline & Abdomen AP & $\mathrm{KAP}$ in $\mu \mathrm{Gy} \cdot \mathrm{m}^{2}$ & 4.5 & 15 & 25 & 47.5 & 70 \\
\hline Fluoroscopy & MCU & $\mathrm{KAP}$ in $\mu \mathrm{Gy} \cdot \mathrm{m}^{2}$ & 30 & 70 & 80 & 75 & \\
\hline \multirow[t]{3}{*}{$\mathrm{CT}$} & Thorax & DLP in mGy.cm & 35 & 50 & 70 & 115 & 200 \\
\hline & & Age band & $<3 m$ & $3 m-1 y r$ & $1-6 y r$ & $>6 y r$ & \\
\hline & Head & DLP in mGy.cm & 300 & 385 & 505 & 650 & \\
\hline
\end{tabular}

Table 2

Reference levels from Table 1 adapted to age bands used in this work.

\begin{tabular}{|c|c|c|c|c|c|c|}
\hline Modality & Examination & & & & & \\
\hline & & Age band & $<1 y r$ & $1-5 y r$ & $5-10 y r$ & $10-16 y r$ \\
\hline & & Corresponding weight band & $<10 \mathrm{~kg}$ & $10-20 \mathrm{~kg}$ & $20-35 \mathrm{~kg}$ & $35-66 \mathrm{~kg}$ \\
\hline \multirow[t]{2}{*}{ Radiography } & Thorax AP/PA & KAP in $\mu$ Gy.m2 & 2.1 & 3.5 & 5.4 & 8.1 \\
\hline & Abdomen AP & KAP in $\mu$ Gy.m2 & 9.6 & 19 & 33 & 59 \\
\hline Fluoroscopy & MCU & KAP in $\mu$ Gy.m2 & 56 & 77 & 78 & \\
\hline \multirow[t]{2}{*}{ CT } & Thoax & DLP in mGy.cm & 42 & 55 & 82 & 156 \\
\hline & Head & DLP in mGy.cm & 385 & 505 & 650 & 650 \\
\hline
\end{tabular}

\subsection{Possible interventions}

There are many factors which will influence optimization of a given imaging task. Some of the common approaches to intervention which should be considered are given in Table 3 for the different modalities. Organizations, such as the International Atomic Energy Agency [24], the European Society of Radiology [25] and the Image Gently Alliance [26] have published more detailed information on optimization and radiation protection of paediatric patients in radiology.

\subsection{Implementation of interventions}

Any intervention in the imaging chain needs to be carried out carefully so as to prevent any adverse impact on the patient during the critical implementation period, particularly variations in image quality. Where possible, the effect of the proposed change should be modelled to provide reassurance that both dose and image quality will be acceptable. Phantom measurements can be used for such purposes, but also

Table 3

Possible interventions in diagnostic radiology.

\begin{tabular}{|c|c|c|}
\hline General Radiography & Fluoroscopy & Computed Tomography \\
\hline Improved collimation & Improved collimation & $\begin{array}{l}\text { Ensuring protocols amended } \\
\text { from adult protocols } \\
\text { according to patient size }\end{array}$ \\
\hline $\begin{array}{l}\text { Revised choice of } \mathrm{kV} \\
\text { depending on size }\end{array}$ & $\begin{array}{l}\text { Revised choice of } \mathrm{kV} \\
\text { and } \mathrm{mA} / \text { Automatic } \\
\text { Brightness Control used }\end{array}$ & $\begin{array}{l}\text { Reduction in the number of } \\
\text { phases used for the standard } \\
\text { examination }\end{array}$ \\
\hline $\begin{array}{l}\text { Revised mAs / AEC } \\
\text { setting (detector } \\
\text { dose setting) used }\end{array}$ & $\begin{array}{l}\text { Selection of increased } \\
\text { filtration }\end{array}$ & $\begin{array}{l}\text { Limiting the extent of the scan } \\
\text { range to the necessary area }\end{array}$ \\
\hline $\begin{array}{l}\text { Selection of increased } \\
\text { filtration }\end{array}$ & $\begin{array}{l}\text { Use of pulsed } \\
\text { fluoroscopy }\end{array}$ & $\begin{array}{l}\text { Appropriate use of iterative } \\
\text { reconstruction algorithms if } \\
\text { available }\end{array}$ \\
\hline $\begin{array}{l}\text { Upgrade of the } \\
\text { detector technology }\end{array}$ & $\begin{array}{l}\text { Shorter fluoroscopy } \\
\text { times }\end{array}$ & $\begin{array}{l}\text { Use of automated mA with } \\
\text { appropriate reference mAs/ } \\
\text { noise level }\end{array}$ \\
\hline $\begin{array}{l}\text { Change in use of grid } \\
\text { and grid type }\end{array}$ & $\begin{array}{l}\text { Reduction in number of } \\
\text { image acquisitions }\end{array}$ & $\begin{array}{l}\text { Adjustment of the } \mathrm{kV} \text { used, to } \\
\text { be appropriate for patient size } \\
\text { and examination type, } \\
\text { preferably using an automatic } \\
\mathrm{kV} \text { selection option } \\
\text { Consistent use of appropriate } \\
\text { field of view and filter } \\
\text { Improved centering/ } \\
\text { positioning of the patient }\end{array}$ \\
\hline
\end{tabular}

modelling the effect of any intervention, as for example by using thinner slice reconstruction to visualize the effect of increased noise, with decreased mAs in CT. For some interventions, training of clinical staff may be required for setting protocols and for interpreting images and, in all cases, clinical staff must be involved in, and supportive of, proposed changes. Where interventions cannot easily be modelled prior to clinical implementation changes in technique should be made in small steps, with checks made on dose and image quality after each step.

Within the CRP, identified interventions were implemented in a variety of ways as discussed in the results section.

\subsection{Re-evaluation of dose and image quality data}

This should be carried out in the same way as described above, and as carried out during the first stage of the study, including using the same personnel for subjective image quality assessment. This stage was completed for a small number of participants in the CRP following implementation of proposed interventions.

\section{Results}

\subsection{Identification of the imaging situation}

The approach taken in the CRP was generally found to work well, with data collection manageable by institutes and data suitable for comparative purposes. Due to the limited paediatric data at some centres, data collation was not limited by clinical indication, and this did raise some uncertainties particularly in comparisons of CT dose data. For example, paediatric head scan for trauma would have a larger DLP compared to a relevant scan for neurological purposes, as it would likely be longer to also cover the C-spine.

The equipment used by participants for radiography included both Computed Radiography (CR) and Digital Radiography (DR) systems, digital systems for fluoroscopy and multidetector CT scanners from 16 to 128 slices.

\subsection{Collection of dose data}

The distributions of examinations reported by all participants, by age group and modality, are illustrated in Fig. 2.

Data were collected in several ways, including manual data entry from displayed equipment parameters, and also automated download from radiology management systems. Data were collated locally then 


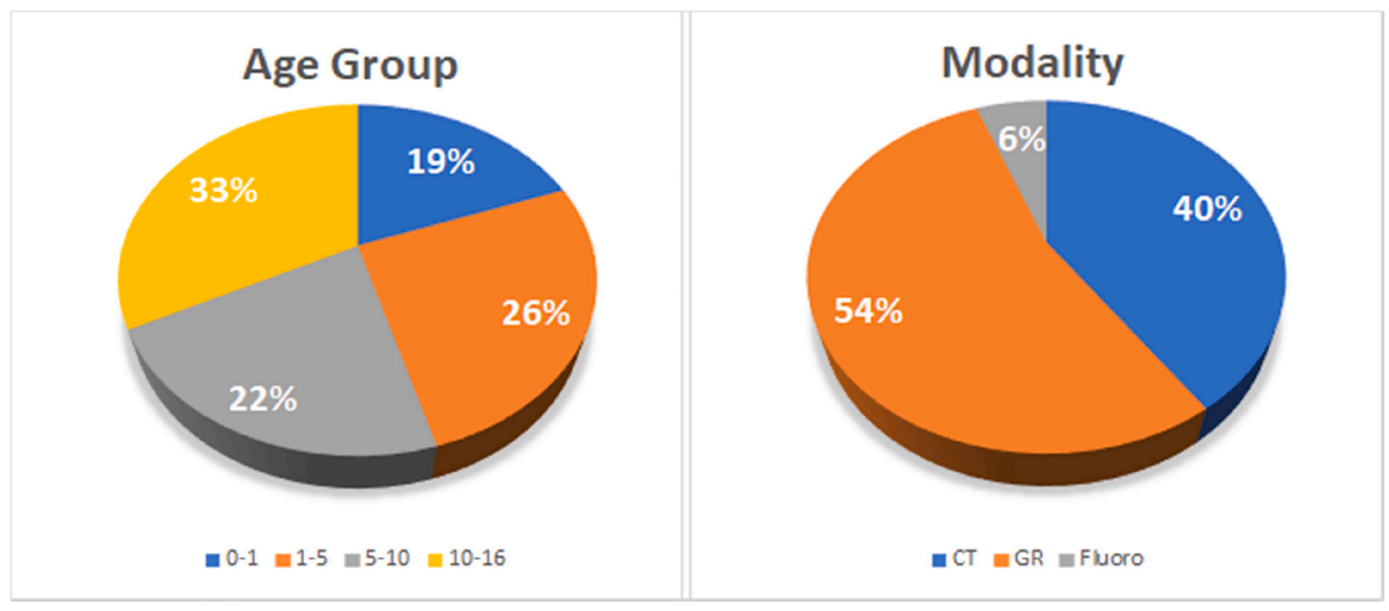

Fig. 2. Distribution of patient data $(\mathrm{N}=1451)$ per age group (a) and per modality (b).

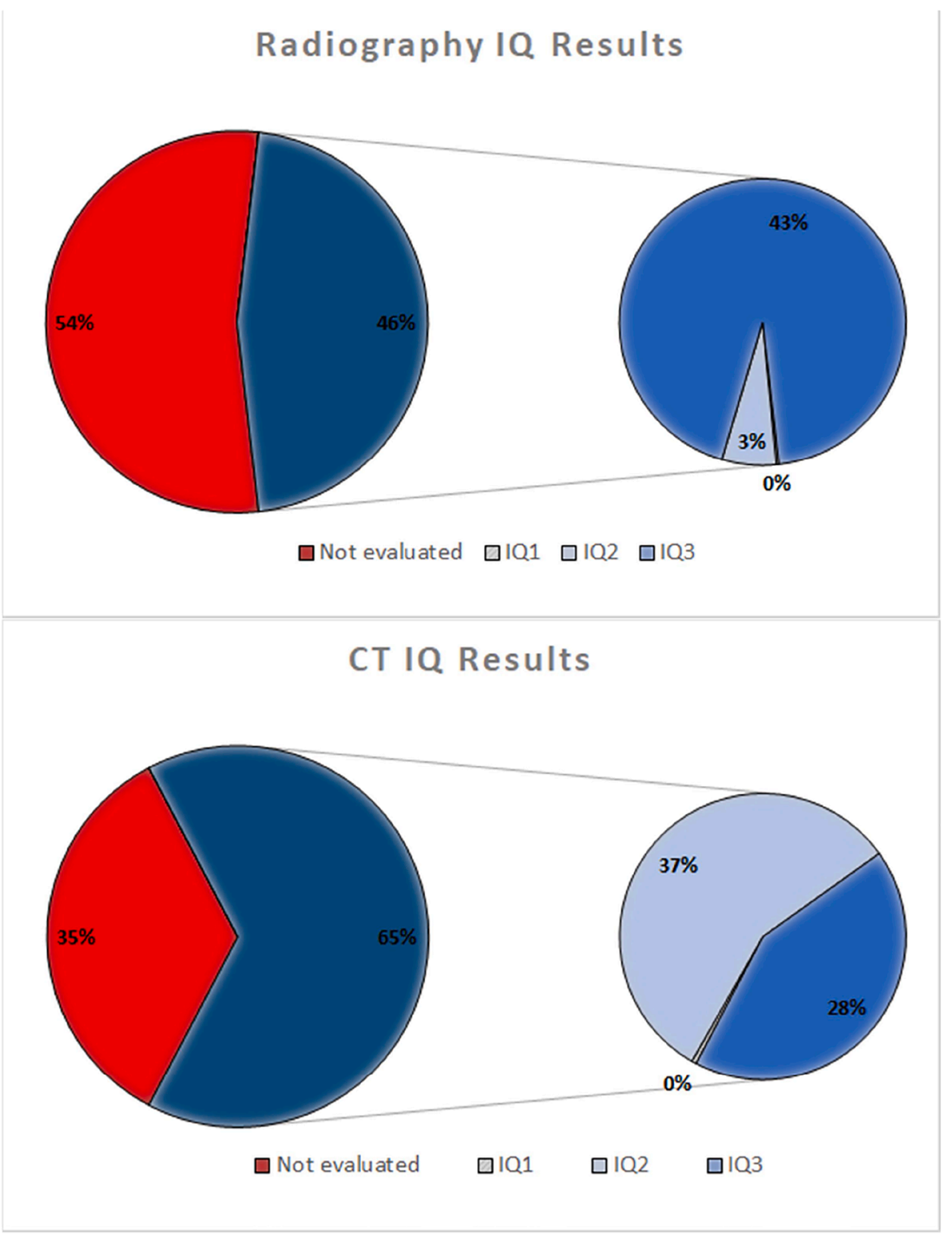

Fig. 3. Percentage of evaluated images in terms of image quality and relevant scores. 
sent to a central coordinator to create a comparative report. Calibration data for each dose quantity and information regarding the measured or displayed units had to be included, albeit these were missing or incomplete in some instances, leading to large uncertainties, even up to one order of magnitude, in associated dose data.

A number of data collection issues were identified, primarily with KAP determination or recording. This included transcription errors in transferring data from one spreadsheet to another; incorrect conversion between KAP units; and inconsistent use of units within a data set. Once identified, these were addressed and corrected.

\subsection{Evaluation of image quality}

Two of the six participating institutes providing dosimetric data from radiology exams were not able to provide data on image quality for individual patient examinations, due to a lack of availability and willingness of the clinicians. It was also evident that one participating centre had initially reported the image quality factor levels in reverse to what was intended (IQ1 best and IQ3 inadequate). This was corrected, and the distribution of image quality scores is shown in Fig. 3.

IQ1, according to the definition of the scale, corresponds to rejected images. Such images were not included in the study. However poor quality, but diagnostic images should by definition be assigned to IQ2. In the final collation of image quality scores submitted by the participants it became apparent that IQ scores had not been interpreted consistently with regard to IQ2 (borderline acceptable) and IQ3 (acceptable).

\subsection{Comparison of dose data}

Data were submitted to a central coordinator, who carried out checks on consistency and raised queries when necessary with the relevant participant. For each modality and age group, dose parameters were compared for the various participating institutes, along with selected technique parameters.

The largest differences in dose levels applied were found for general radiography thorax examinations with a factor of over one order of magnitude between the lowest and the highest average patient dose. This is also reflected by a coefficient of variation (CoV) between center mean values ranging from 0.72 ( $5-10 \mathrm{yr}$ olds) to 0.94 ( $0-1 \mathrm{yr}$ olds) with a mean value of 0.82 . These data are shown in Table 4 and Fig. 4 . Since no statistically significant difference was found in thorax X-rays taken
AP versus PA in the same age group and institution, AP and PA were pooled. Country A reported the values collected for the smallest two age groups as implausible because of a system malfunction that led to putting the system out of operation shortly after this study. Fig. 4a shows KAP values reported by the participants. Considering the results reported by all centres, one can notice a skewness, which can be attributed to poor optimization in certain cases. In Fig. 4b KAP data is shown relative to DRLs from Table 2. A logarithmic presentation was chosen to better demonstrate variance of doses reported by participants. Although the results cannot be directly compared with the DRL values, due to the different technologies used and the patient habitus, they can be used as an indication of the level of exposure for different institutes.

For CT protocols, the reported DLP values were more homogeneous, with differences in average DLP values up to a factor of around three, as illustrated for CT brain examinations in Table 5 and Fig. 5. Between centers the $\mathrm{CoV}$ averaged over all age group was 0.39 (minimum 0.27 for $5-10 y r$, maximum 0.56 for $10-16 y r$ ). This indicates a much smaller dose variation compared to those reported in other studies $[27,28]$. The careful examination of the boxplots shown in Figs. 4 and 5 can emphasize opportunities for optimizing practices and review clinical protocols, as will be discussed below.

\subsection{Identification of interventions}

Within the CRP, a number of interventions were identified, in response to the data comparison exercise. In one institute, high doses in GR were associated with use of collimation to detector rather than anatomical area (excessive field sizes). The use of a low $\mathrm{kV}$ technique, even for the oldest age-group, was also identified, and therefore a strategy of increasing $\mathrm{kV}$ and reducing $\mathrm{mAs}$ was indicated, along with training of clinical staff in the effective use of collimation.

For CT, two participants identified that high doses were due to adult protocols being used for all paediatric patients. One institute raised an issue with image quality for paediatric thorax CT, in that noise levels within soft tissue of CT thorax scans for smaller sized patients were felt to be too high for reliable diagnosis. As the dose values obtained were substantially lower than those of other participants and, for smallest patients, an order of magnitude below the DRLs given in Tables 1 and 2, a need to increase the reference mAs value used for dose modulation was identified. The factor between doses at this centre and DRLs reduced with increasing patient size, to a factor of around 2 for $10-15$ yrs. In

Table 4

Statistical evaluation of KAP values $\left(\mu \mathrm{Gy} \cdot \mathrm{m}^{2}\right)$ provided by participants for Thorax X-rays. \%AP indicates percentage of thorax X-rays having been taken AP. *: Numbers in italic (participant A) have been reported as questionable after submission due to system malfunction.

\begin{tabular}{|c|c|c|c|c|c|c|c|c|c|}
\hline & Age Group & $\mathrm{N}$ & $\% \mathrm{AP}$ & Mean & STD & $\mathrm{CoV}$ & Median & 3rd Quartile & IQR \\
\hline \multirow[t]{4}{*}{ A } & $0-1 y r$ & 6 & $100 \%$ & $0.08^{*}$ & $0.10^{*}$ & $1.17^{*}$ & $0.04 *$ & $0.16^{*}$ & $0.14^{*}$ \\
\hline & $1-5 y r$ & 10 & $100 \%$ & $0.37^{*}$ & $0.40^{*}$ & $1.06^{*}$ & $0.22 *$ & $0.64 *$ & $0.55^{*}$ \\
\hline & $5-10 \mathrm{yr}$ & 6 & $0 \%$ & 3.22 & 2.40 & 0.75 & 2.62 & 5.32 & 4.07 \\
\hline & $10-16 y r$ & 12 & $0 \%$ & 5.73 & 2.20 & 0.38 & 5.30 & 6.68 & 2.30 \\
\hline \multirow[t]{4}{*}{ B1 } & $0-1 \mathrm{yr}$ & 6 & $67 \%$ & 0.44 & 0.28 & 0.64 & 0.46 & 0.68 & 0.51 \\
\hline & $1-5 y r$ & 25 & $96 \%$ & 0.30 & 0.14 & 0.47 & 0.26 & 0.36 & 0.15 \\
\hline & $5-10 y r$ & 26 & $69 \%$ & 0.72 & 0.61 & 0.84 & 0.61 & 0.85 & 0.49 \\
\hline & $10-16 y r$ & 79 & $43 \%$ & 1.33 & 0.83 & 0.62 & 1.06 & 1.66 & 0.95 \\
\hline \multirow[t]{4}{*}{ B2 } & $0-1 \mathrm{yr}$ & 0 & & & & & & & \\
\hline & $1-5 y r$ & 46 & $83 \%$ & 2.14 & 1.36 & 0.63 & 1.70 & 2.33 & 0.93 \\
\hline & $5-10 y r$ & 66 & $42 \%$ & 3.22 & 1.36 & 0.42 & 2.93 & 3.88 & 1.68 \\
\hline & $10-16 y r$ & 172 & $13 \%$ & 4.89 & 2.04 & 0.42 & 4.46 & 5.35 & 1.53 \\
\hline \multirow[t]{4}{*}{$\mathrm{C}$} & $0-1 y r$ & 34 & $100 \%$ & 3.34 & 2.47 & 0.74 & 2.52 & 5.61 & 4.23 \\
\hline & $1-5 y r$ & 22 & $100 \%$ & 4.26 & 2.54 & 0.60 & 4.53 & 5.36 & 3.45 \\
\hline & $5-10 y r$ & 16 & $75 \%$ & 7.04 & 5.63 & 0.80 & 4.63 & 8.20 & 4.47 \\
\hline & $10-16 y r$ & 9 & $11 \%$ & 13.60 & 5.35 & 0.39 & 12.13 & 18.20 & 8.88 \\
\hline \multirow[t]{4}{*}{$\mathrm{E}$} & $0-1 y r$ & 33 & $100 \%$ & 1.08 & 0.42 & 0.38 & 1.02 & 1.39 & 0.67 \\
\hline & $1-5 y r$ & 35 & $94 \%$ & 1.51 & 0.81 & 0.54 & 1.18 & 1.88 & 0.90 \\
\hline & $5-10 \mathrm{yr}$ & 26 & $15 \%$ & 2.16 & 0.78 & 0.36 & 2.13 & 2.64 & 1.17 \\
\hline & $10-16 y r$ & 24 & $0 \%$ & 3.57 & 1.57 & 0.44 & 3.50 & 4.41 & 2.08 \\
\hline
\end{tabular}


a)

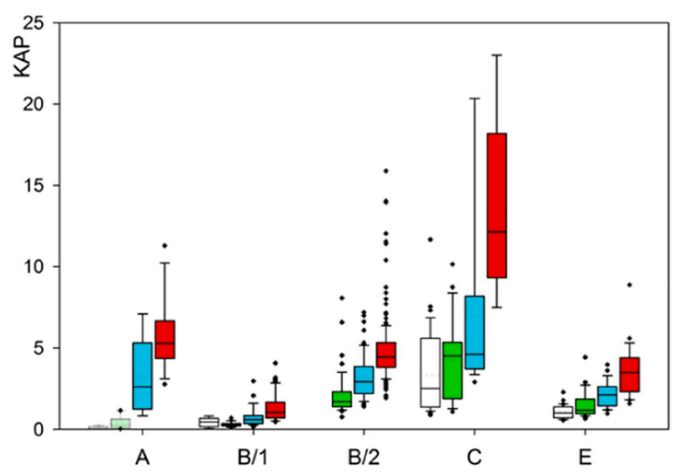

b)

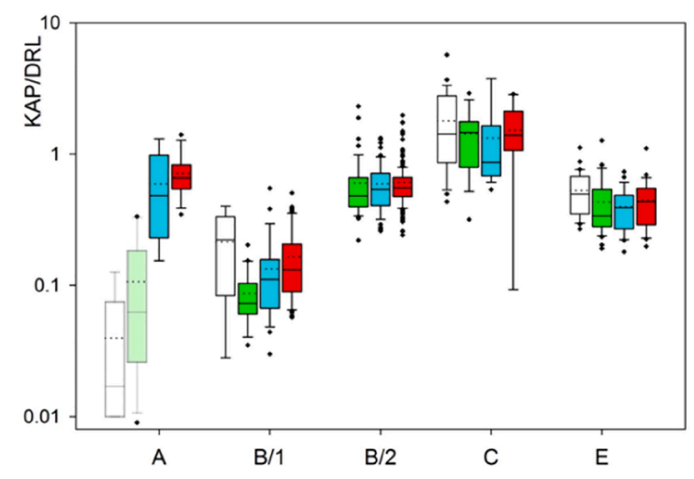

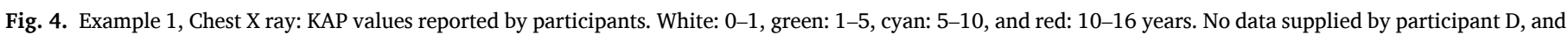

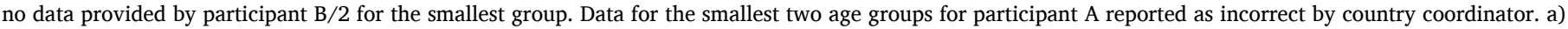

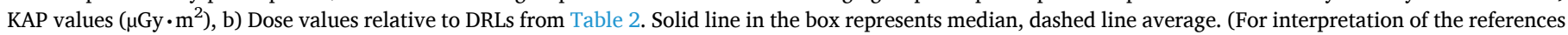
to colour in this figure legend, the reader is referred to the web version of this article.)

Table 5

Statistical evaluation of DLP $(\mathrm{mGy} \cdot \mathrm{cm})$ from original Brain CT data provided by the participants.

\begin{tabular}{|c|c|c|c|c|c|c|c|c|}
\hline & $\begin{array}{l}\text { Age } \\
\text { Group }\end{array}$ & $\mathrm{N}$ & Mean & STD & $\mathrm{CoV}$ & Median & $\begin{array}{l}\text { 3rd } \\
\text { Quartile }\end{array}$ & IQR \\
\hline \multirow[t]{4}{*}{ A } & $0-1 \mathrm{a}$ & 12 & 263 & 105 & 0.40 & 195 & 393 & 211 \\
\hline & $1-5 a$ & 16 & 414 & 48 & 0.12 & 401 & 465 & 90 \\
\hline & $5-10 a$ & 7 & 519 & 43 & 0.08 & 525 & 567 & 97 \\
\hline & $10-16 a$ & 4 & 621 & 29 & 0.05 & 624 & 647 & 54 \\
\hline \multirow[t]{4}{*}{ B } & $0-1 \mathrm{a}$ & 20 & 471 & 133 & 0.28 & 437 & 539 & 158 \\
\hline & $1-5 a$ & 20 & 557 & 139 & 0.25 & 563 & 638 & 120 \\
\hline & $5-10 a$ & 20 & 578 & 113 & 0.20 & 552 & 649 & 156 \\
\hline & $10-16 a$ & 20 & 632 & 77 & 0.12 & 649 & 649 & 108 \\
\hline \multirow[t]{4}{*}{ C } & $0-1 \mathrm{a}$ & 24 & 603 & 203 & 0.34 & 593 & 695 & 249 \\
\hline & $1-5 a$ & 28 & 786 & 374 & 0.48 & 742 & 921 & 407 \\
\hline & $5-10 a$ & 15 & 765 & 239 & 0.31 & 783 & 962 & 452 \\
\hline & $10-16 a$ & 9 & 1704 & 637 & 0.37 & 2016 & 2194 & 1249 \\
\hline \multirow[t]{4}{*}{ D } & $0-1 \mathrm{a}$ & 52 & 205 & 77 & 0.38 & 214 & 266 & 123 \\
\hline & $1-5 a$ & 33 & 360 & 168 & 0.47 & 326 & 497 & 221 \\
\hline & $5-10 a$ & 25 & 469 & 126 & 0.27 & 463 & 529 & 123 \\
\hline & $10-16 a$ & 24 & 554 & 191 & 0.34 & 517 & 671 & 233 \\
\hline \multirow[t]{4}{*}{$\mathrm{E}$} & $0-1 \mathrm{a}$ & 3 & 411 & 79 & 0.19 & 440 & 472 & 151 \\
\hline & $1-5 a$ & 21 & 520 & 112 & 0.21 & 556 & 587 & 68 \\
\hline & $5-10 a$ & 13 & 869 & 164 & 0.19 & 919 & 923 & 92 \\
\hline & $10-16 a$ & 20 & 795 & 108 & 0.14 & 762 & 861 & 138 \\
\hline
\end{tabular}

a)

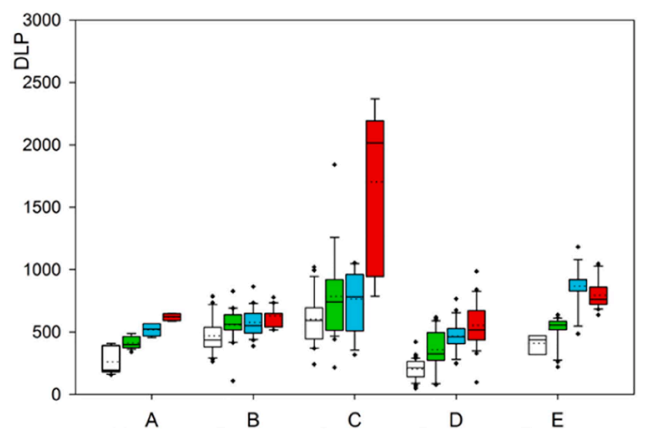

another institute, unnecessary large DLPs were due to unnecessary long scans for brain scans (192 $\mathrm{mm}$ in newborns on average, to $251 \mathrm{~mm}$ in 10-16-year olds).

\subsection{Implementation of interventions}

At one institute, changes to CT protocols were made in agreement with the clinical staff. Initially, a comparative study considering the current thorax and brain protocols was carried out, adopting similar studies published by the American Association of Physicists in Medicine (AAPM) as a reference [29]. In sequence, the chief radiologist and a qualified radiographer checked the proposed optimized protocols and suggested some changes, including adoption of lower mAs than those proposed by the AAPM. The next step was the combined action of the medical physicist and the medical radiology technologists in order to adapt the age-groups for the considered protocols and to organize their selection in the CT console and instruct the users. Then, the team of medical radiology technologists were trained on the use of the new protocols. It is important to emphasize that changes on the display of the machine console were useful to differentiate paediatric from adult protocols and also to simplify the choice of the correct age-group by the user. A simple colour-code was adapted for this purpose. Only after these steps the new protocols were implemented in the clinical routine. Fig. 6 shows a flow chart relating the steps conducted for the optimization process of paediatric thorax and brain CT. During this period, the opinion of the radiology staff was monitored in order to identify questions or complaints associated to loss of image quality or increased noise. No negative observation was identified, which was associated with an adequate acceptance of this new image quality reference by the local

b)

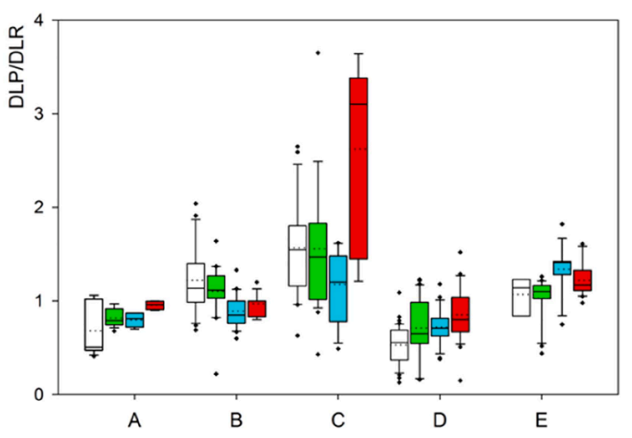

Fig. 5. Example 2. Head CT: DLP values reported by participants. Age group colours as in Fig. 1. a) DLP values in mGy $\mathrm{cm}$, b) DLP values relative to DRLs from Table 2. Solid line: median, dashed line: average. 


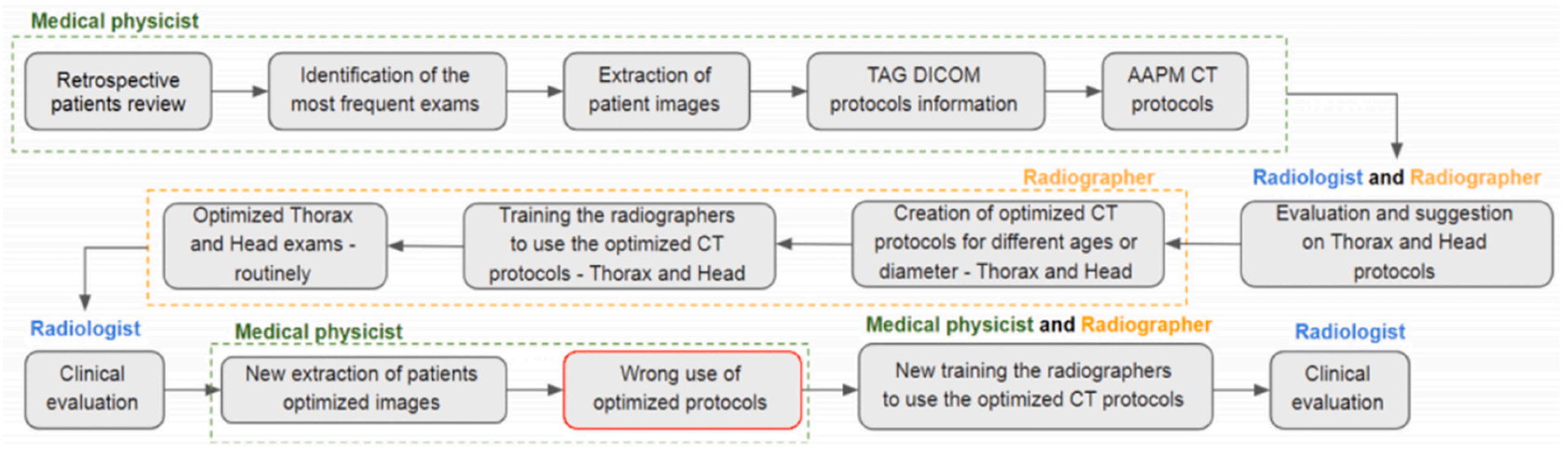

Fig. 6. Flow chart relating the steps conducted for the optimization process of paediatric thorax and brain CT by one of the participating institutes [30].

radiologists.

\subsection{Re-evaluation of dose and image quality data}

The final stage in the optimization cycle was completed by 2 participants for 3 examination types, namely brain and thorax CT for one and thorax CT for the other. Re-assessment of data at the same institute using the same personnel and methodology is inherently more reliable than comparisons between centres, provided Quality Control tests have been continued.

The impact of the implementation of the new CT protocols for participant B is shown in Fig. 7. Comparison with DRLs (Fig. 7b) demonstrates that before optimization relative doses especially to the smaller children were higher than necessary, while after optimization dose levels relative to DRLs are (nearly) independent of age group.

During the comparative analysis of the thorax CT results, centre B demonstrated substantially lower dose values than other centres. Following this finding, clinical assessment of image quality for these scans was performed locally and revealed that images were only borderline acceptable and, in some instances, at risk of being nondiagnostic (image quality grade: IQ2). A multi-professional team comprising of a medical physicist, medical imaging technologist and radiologist met to review protocols. Reference mAs being used for each paediatric protocol were doubled in combination with a decrease in $\mathrm{kV}$, demonstrating that optimization is not always equivalent to dose reduction. The protocol changes were made on the scanner console and stored within the equipment database. There were no changes made to the naming of the protocols or operator procedures. Following the changes in the protocols DLP readings and diagnostic image quality of the resulting patient images were closely monitored to ensure clinical acceptability by the radiologists. The results for the optimisation of CT thorax protocols for centre B are given in Fig. 8. Although the number of data initially collected after optimization is low, doses seem to have slightly increased, but are still roughly within the interquartile ranges (IQR) derived before optimization. Image quality was improved noticeably to give consistently clinically acceptable images (image quality grade: IQ3). Delivered doses became more consistent with lower IQR. However, since doses are actually still exceeding the reference values, it would be advisable to continue with another optimization round after staff has become acquainted to the new settings and the resulting diagnostic image quality. This example highlights that optimization is a slow and continuous process.

\section{Discussion}

Participants in the CRP have successfully engaged in and reviewed optimization processes for paediatric imaging procedures, using the steps outlined here. However, obstacles were identified for four of the five participants. Each step has been proven important and cannot be missed, so optimization may be a relatively slow process, particularly for less frequently performed procedures. Normally, a time frame of a few months should be allocated, although in certain cases during this study, the complete process could not be completed even within the allocated frame of the CRP, which corresponded to 1.5 years. The greatest problems experienced lay in correctly collecting dose data, obtaining image quality data and, in some cases, difficulty persuading clinicians to make changes to established practices identified as suboptimal. The latter issue was especially evident if the optimization procedure was not initiated and carried out internally with involvement of all relevant professional groups (radiologists, medical physicists and medical radiology technologists). However, since data collection errors were in many cases not detected internally, actively seeking advice from external experts has been proven advantageous. This is also true, for the whole optimization process, when departments are firstly engaged into a)

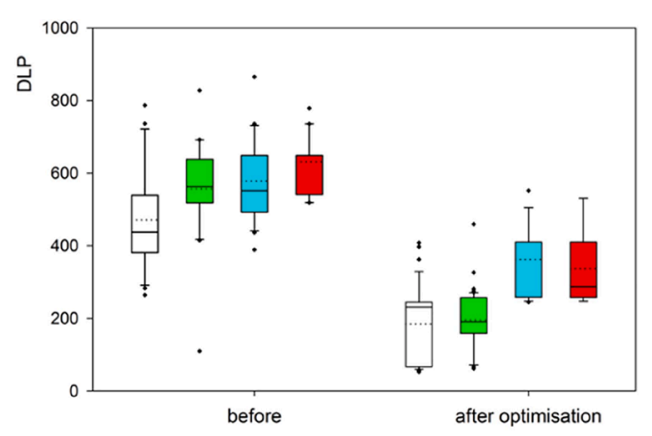

b)

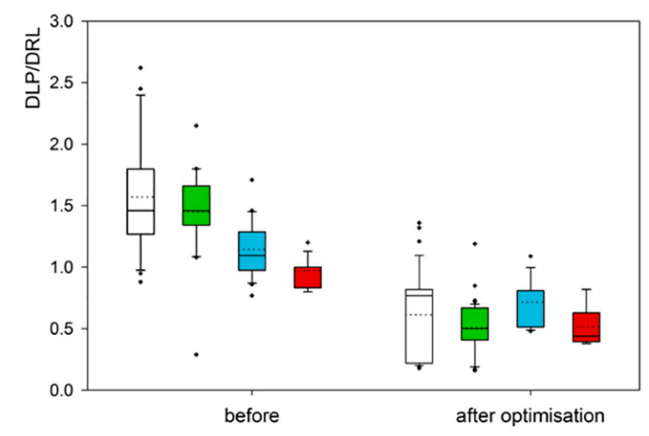

Fig. 7. Comparative results of the optimized and non-optimizes DLPs brain CT scans in Centre B. a) DLP values (mGy $\cdot \mathrm{cm}$ ), b) DLP values divided by DRLs from Table 2. Colours representing age groups as in Fig. 4. Number of data before optimization is 20/group and after optimization is $35,63,13,8$ for smallest to largest age groups, respectively. Solid line: median, dashed line: average. 

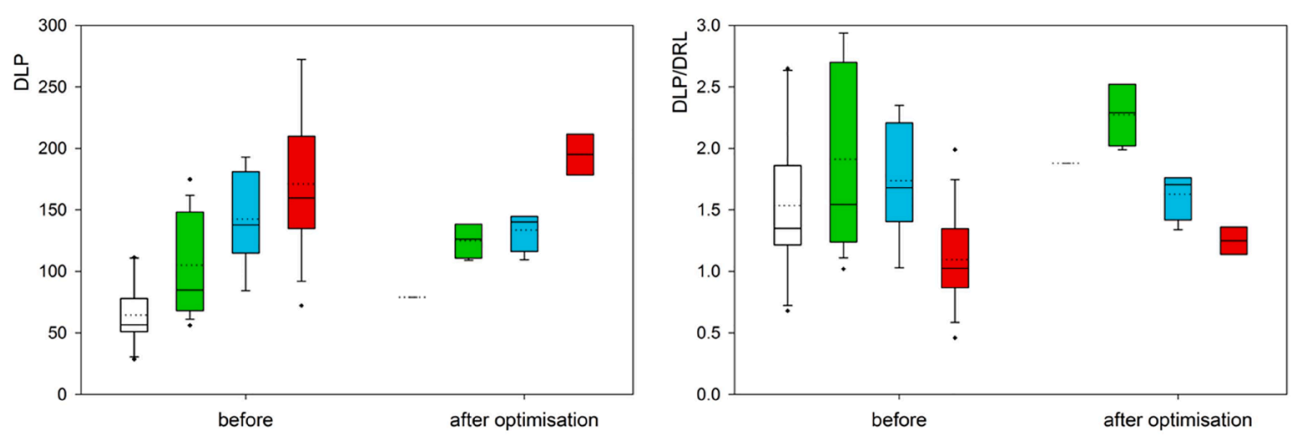

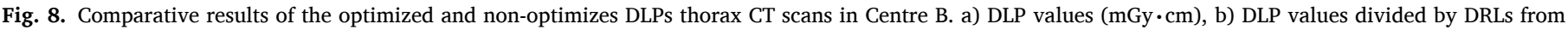

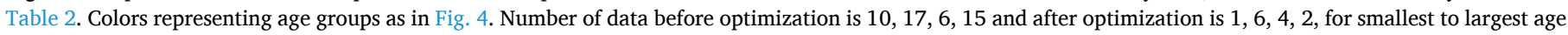
groups, respectively. Solid line: median, dashed line: average.

relevant exercises, when expertise on the process is poor. Also international or professional organizations can play an important role [31]. Especially in the field of imaging medical physics, which is often lacking, collaboration with external experts or collaborative activities between centres can maximize the efficacy and benefits of the process.

The experiences of participants in the CRP reflect those frequently illustrated within published studies and, in particular, multi-centre evaluation and optimization activities. The fundamental issue was that in order to effectively carry out the comparison stage, as a pre-requisite to identifying and implementing optimization strategies, it was necessary to have a high degree of confidence in the data obtained. There are, however, a number of reasons why such confidence may be lacking, in relation to both dosimetric and image quality parameters, as discussed below.

Any instance of transcribing information carries a risk of manual error. Such errors are likely to be fairly random in nature, and likely to give rise to individual data outliers, although more systematic errors may be made if information is misinterpreted and transcribed into an incorrectly headed data column for instance. The likelihood of the former circumstance may be reduced by due care and attention, plus additional data checking, and the second by improved education or instruction. However, both may be difficult to identify when analysing data remotely, when access to the original raw data is not possible.

Electronically transferred data has the advantage of preventing human transcription errors, however, there remain several opportunities for errors to occur. The most probable of these arises from the continued lack of standardisation of dosimetry quantities. For international studies, the use of both SI and non-SI units is common and, for some dose quantities such as air Kerma-Area-Product (KAP) there are a number of equivalent SI units in popular use. Different pieces of imaging equipment may use different standard units for displaying and transferring dose data, and data management systems may have different approaches to interpreting and storing dose units. For this reason, data may easily be presented in error by a factor of 10 or 100 . Another issue of electronically transferred and automatically collected dose data seen in this project is that users tend to assume its validity and accuracy to a higher degree as is the case with measured data, and thus may be less inclined to question it. In one case (centre A, youngest 2 age groups) KAP values from a CR device were obviously wrong, most likely because of an error in data interfacing. However, this issue has persistently been present, despite a quality system being in place involving both manufacturer and local medical physicist periodically checking all equipment in an ISO 9001 quality management certified institute.

A further source of uncertainty in dosimetry data, that may potentially give rise to a large systematic error, is the lack of reliable calibration data for the dose monitoring equipment or electronic display. Calibration methodologies for KAP meters and dosemeters used for output measurements are well established, but the extension of these to integral displayed parameters is less well motivated. As a minimum, the accuracy of displayed dose parameters should be checked during commissioning and quality assurance of equipment and corrected if outside an acceptable tolerance level. Additionally, when the used metric is based on CTDI values provided on the DICOM header or dose report, it has to be confirmed that the reference phantom size adopted is consistent to the patient size or anatomical region of the protocol being investigated. Otherwise, wrong information can be registered and, if not noted, inadequate interventions can be introduced on the CT equipment or protocols.

For a single centre analysing data, it may be relatively easy for staff with appropriate experience and access to raw data to assess and address the impact of the various factors described above but, once data is sent further afield to be collated at a regional, national, or international level, it may be impossible to make such data quality assessments. Similarly, it is often difficult to judge the quality and reliability of published data for the same reason.

Children were grouped in age bands for data evaluation and interpretation. However, weight and height were also obtained wherever possible to ensure data used in analysis was from children with standard (typical, average) body habitus. Using weight bands, or more advanced indices such as the patient diameter, rather than age bands would possibly reduce the data variability within each group, but would increase the complexity of the data collection process, leading to reduced compliance. In this coordinated research project, data from standard patients undergoing standard procedures that were defined in the first project step were collected, excluding exceptionally over- or underweight children.

In the optimization process, the comparison of the findings regarding dose levels, image quality and imaging parameters used with corresponding ones from similar practices (either through multicentre collaboration or literature review) is most valuable for the progression of the optimization process. However, in cases where this is not possible, (as in the use of very specialized techniques, or lack of comparable data, e.g.), even the review of the internally collected data can lead to improvements. The evaluation of image quality and the technique factors have, in this case, particular potential to reveal weaknesses (e.g. use of incorrect field sizes or scan length, unnecessary high pulse rate) that can further assist continuous improvement within a department.

Regarding data comparison, it is important to be aware that imaging below reference dose levels does not necessarily indicate appropriate procedures. Especially in cases when doses are very low [32] this should initiate further evaluation, particularly regarding the image quality and the diagnostic content of the resulted examinations. During this study, this was especially evident in one institute where MCUs were performed, with KAP values of approximately half of the DRLs, with automatic brightness control compensating for attenuation of the contrasted filled bladder resulting in extremely overexposed images. Low dose levels were due to short fluoroscopy time, appropriately low frame rates and detector dose level, and high added filtration. In this case these images 
were graded as image quality IQ3 and no optimization was performed. This issue emphasises the need for appropriate training for all specialists involved, medical radiology technologists, medical physicists and radiologists. In other cases, apparently low dose readings were found to be incorrect because of either missing or wrong calibration or an issue with data interfacing. These had not been followed up locally because doses did not appear to be at a level necessitating intervention. Issues with too low image quality due to very low dose levels have not been commonly found in this CRP. However, in one institution (E) having reported very low dose levels in paediatric CT, the image quality was felt to be insufficient due to noise in the soft tissue regions obscuring the low contrast, particularly for smallest patients. The reference mAs, and thus dose, was increased in this institution.

A well structed optimization strategy was followed in this work. Whereas the definition of people involved (the "who") was straight forward in all centres, difficulties were seen in the time frame (the "when") and the "how". The latter two were not completely achieved in some institutions having supplied data but falling short of analyzing it appropriately. The assessment of diagnostic image quality was identified as a major issue, as well as the definition and implementation of procedural changes. Regarding the former, two of five participants could not collect image quality data most likely due to lack of input from radiologists to this part of the project. The image quality grading scheme has been interpreted in different ways by some participants with regard to IQ2 and IQ3. IQ2 "borderline acceptable", and IQ3 ("acceptable") were perceived potentially differently, as "acceptable" and "excellent" respectively. At least one institution understood "excellent" in this respect, grading low dose images showing appropriate noise levels as "excellent". In addition to an additional IQ level clearly and unambiguously indicating images having been acquired with better-thannecessary IQ for future optimization studies of this nature, greater standardisation of subjective image quality requirements would be of great value. In this sense the use of a 5-level scale, as proposed in the literature for relevant studies $[33,34]$ would be beneficial as it can better demonstrate the quality of the acquired images, including those with image quality unnecessary high for the diagnostic task. This would require the commitment of the clinical staff participating in this optimization exercise, since it would increase the complexity and thus the time required for the evaluation of the images. Nevertheless, the results from this coordinated research project suggest that the added complexity of the image quality assessment using a 5-point rather than a simple 3-point Likert scale in image quality evaluation should outweigh the putative simplicity of using a 3 point scale.

In addition, retrospective and also prospective evaluation of diagnostic image quality performed by local staff will be potentially biased by either personnel preferences or level of experience and expertise. Image quality auditing by external experts was not available from this study design. It is worth mentioning that one institute using inappropriate technique factors did neither evaluate image quality locally, nor ask for assistance.

\section{Conclusion and recommendations}

The experience of the CRP demonstrated both the practicality and benefits of previously published strategies for optimization, as applied to paediatric diagnostic radiology examinations, and the issues that commonly arise during such activities which may have a detrimental effect on the results. This has led to identification of key points that require close attention in order to achieve successful practical outcomes in optimization. These are summarised in Table 6 as 'Ten Practical Tips' for optimization.

In addition, a number of successful interventions have been carried out to optimize dose and image quality for paediatric examinations, using the methodologies outlined. These include development of age/ size specific CT protocols in some institutes and improved image quality with slightly increased doses for paediatric CT in another institute.
Table 6

Ten practical tips for optimization.

1 Inclusion - involve medical physicist, radiographer and radiologist.Seek external expertise and advice if unsure

2 Calibration - check accuracy and tolerance of displayed dose parameters

3 Recording and archiving data for later review - initially keep exactly as displayed or downloaded

4 Data accuracy - check at each stage of automated data transfer, in case of manual transcription of data - get it independently checked

5 Unit conversion/calibration coefficients - get them independently checked

6 Comparison of data - consider uncertainties

7 Evaluate image quality - encourage clinicians to participate and use an appropriate IQ grading scale

8 Interventions - ensure clinical acceptability via phantom tests and/or gradual introduction

9 Implementation - provide training to clinical staff \& ensure this step occurs

10 Results - share with all involved staff

These interventions illustrate that, when carried out carefully, with attention to the practicalities raised here, optimization is a valuable tool in improving the safety and quality of diagnostic paediatric examinations.

\section{Acknowledgements}

The authors would like to acknowledge the support of Ms Djarwani Soejoko, Ms Vivian H. Guerra, Ms Denise Y. Nersissian, Mr Ahmed Gharieb, Mr Sayed Abdou and Mr Shirazu Issahaku for contributing in data collection and analysis at different stages of the study.

This work has been supported by the International Atomic Energy Agency, as part of the CRP E2.40.20 on "Evaluation and Optimization of Paediatric Imaging".

\section{References}

[1] International Atomic Energy Agency. Radiation Protection and Safety of Radiation Sources: International Basic Safety Standards, IAEA Safety Standards Series No. GSR Part 3. Vienna: International Atomic Energy Agency; 2014.

[2] International Atomic Energy Agency and World Health O [accessed 13 F 2020]. Bonn Call for Action 2012. https://www.iaea.org/sites/default/files/17/12/bonncall-for-action.pdf (accessed February 13, 2020).

[3] González L, Vañó E, Ruiz MJ. Radiation doses to paediatric patients undergoing micturating cystourethrography examinations and potential reduction by radiation protection optimization. Br J Radiol 1995;68(807):291-5. https://doi.org/ 10.1259/0007-1285-68-807-291.

[4] Pace E, Borg M. Optimization of a paediatric CT brain protocol: a figure-of-merit approach. Radiat Prot Dosimetry 2018;182:394-404. https://doi.org/10.1093/ rpd/ncy078.

[5] Dougeni E, Faulkner K, Panayiotakis G. A review of patient dose and optimisation methods in adult and paediatric CT scanning. Eur J Radiol 2012;81(4):e665-83. https://doi.org/10.1016/j.ejrad.2011.05.025.

[6] Kiljunen T, Järvinen H, Savolainen S. Diagnostic reference levels for thorax X-ray examinations of paediatric patients. Br J Radiol 2007;80(954):452-9. https://doi. org/10.1259/bjr/60918774.

[7] Dougeni ED, Delis HB, Karatza AA, Kalogeropoulou CP, Skiadopoulos SG, Mantagos SP, et al. Dose and image quality optimization in neonatal radiography. Br J Radiol 2007;80(958):807-15. https://doi.org/10.1259/bjr/77948690.

[8] Mooney R, Thomas PS. Dose reduction in a paediatric X-ray department following optimization of radiographic technique. Br J Radiol 1998;71(848):852-60. https:// doi.org/10.1259/bjr.71.848.9828798.

[9] International Atomic Energy Agency. Dosimetry in Diagnostic Radiology for Paediatric Patients, IAEA Human Health Series No. 24. Vienna: 2013.

[10] Salama DH, Vassileva J, Mahdaly G, Shawki M, Salama A, Gilley D, et al. Establishing national diagnostic reference levels (DRLs) for computed tomography in Egypt. Phys Med 2017;39:16-24. https://doi.org/10.1016/j.ejmp.2017.05.050.

[11] Mohammed Ali A, Hogg P, England A. Dose optimisation in paediatric radiography - Using regression models to investigate the relative impact of acquisition factors on image quality and radiation dose. Phys Medica 2019;68:61-8. https://doi.org/ 10.1016/j.ejmp.2019.10.034.

[12] Tsapaki V, Aldrich JE, Sharma R, Staniszewska MA, Krisanachinda A, Rehani M, et al. Dose reduction in CT while maintaining diagnostic confidence: diagnostic reference levels at routine head, chest, and abdominal CT - IAEA-coordinated research project. Radiology 2006;240(3):828-34. https://doi.org/10.1148/ radiol.2403050993.

[13] Giansante L, Martins JC, Nersissian DY, Kiers KC, Kay FU, Sawamura MVY, et al. Organ doses evaluation for chest computed tomography procedures with TL dosimeters: Comparison with Monte Carlo simulations. J Appl Clin Med Phys 2019; 
20(1):308-20. https://doi.org/10.1002/acm2.2019.20.issue-110.1002/ acm2.12505.

[14] Lee C, Kim KP, Bolch WE, Moroz BE, Folio L. NCICT: A computational solution to estimate organ doses for pediatric and adult patients undergoing CT scans. J Radiol Prot 2015;35(4):891-909. https://doi.org/10.1088/0952-4746/35/4/891.

[15] Seibert JA, Boone JM, Wootton-Gorges SL, Lamba R. Dose is not always what it seems: where very misleading values can result from volume CT dose index and dose length product. J Am Coll Radiol 2014;11(3):233-7. https://doi.org/ 10.1016/j.jacr.2013.10.010.

[16] Poli GL, Coca MA, Torres LA, Fahey FH, Lassmann MH, Chapple CL, et al. Developing and implementing an imaging optimization study in pediatric nuclear medicine: experience and recommendations from an IAEA Coordinated Research Project. J Nucl Med 2020. https://doi.org/10.2967/jnumed.120.244616.

[17] Poli GL, Torres L, Coca M, Veselinovic M, Lassmann M, Delis H, et al. Paediatric nuclear medicine practice: an international survey by the IAEA. Eur J Nucl Med Mol Imaging 2020;47(6):1552-63. https://doi.org/10.1007/s00259-019-04624-w.

[18] International Commission for Radiological Protection. Diagnostic reference levels in medical imaging. ICRP Publication 135. Ann. ICRP 46(1). 2017.

[19] Vassileva J, Rehani M. Patient grouping for dose surveys and establishmentof diagnostic reference levels in paediatric computed tomography. Radiat Prot Dosimetry 2015;165(1-4):81-5. https://doi.org/10.1093/rpd/ncv113.

[20] International Atomic Energy Agency. Dosimetry in Diagnostic Radiology: An International Code of Practice, Technical Reports Series No. 457. Vienna: 2007.

[21] International Atomic Energy Agency. Implementation of the International Code of Practice on Dosimetry in Diagnostic Radiology (TRS 457): Review of Test Results, IAEA Human Health Report, No. 4,. Vienna: 2011.

[22] European Commission. Radiation Protection No 185 - European Guidelines on Diagnostic Reference Levels for Paediatric Imaging. Luxembourg: 2018.

[23] Rodd C, Metzger DL, Sharma A, Cummings E, Chanoine JP, Lawrence S, et al. Extending World Health Organization weight-for-age reference curves to older children. BMC Pediatr 2014. https://doi.org/10.1186/1471-2431-14-32.
[24] International Atomic Energy Agency. Radiation protection of children in radiology. Padiation Prot Patients 2020: https://www.iaea.org/resources/rpop/health -profess.

[25] European Society of Radiology. Paediatric Imaging. Eurosafe Imaging 2020:http:// www.eurosafeimaging.org/ask-eurosafe-imagin.

[26] Image Gently. The Image Gently Alliance 2020: https://www.imagegently.org/.

[27] Rehani MM. Multi-national findings on radiation protection of children. Pediatr Radiol 2014;44(S3):475-8. https://doi.org/10.1007/s00247-014-3125-7.

[28] Vassileva J, Rehani MM, Applegate K, Ahmed NA, Al-Dhuhli H, Al-Naemi HM IAEA survey of paediatric computed tomography practice in 40 countries in Asia, Europe, Latin America and Africa: procedures and protocols. Eur Radiol 2013;23 (3):623-31. https://doi.org/10.1007/s00330-012-2639-3.

[29] American Association of Physicists in Medicine. The alliance for quality computed tomography. 2017 n.d. https://www.aapm.org/pubs/CTProtocols/?tab=5\#CTabb edPanels.

[30] Guerra VH, Nersissian DY, Melo CS, Vasconcellos CECEC, Freitas RG, Sawamura MVY, et al. Pediatric computed tomography dose optimization in a general hospital. Rev Bras Física Médica 2019;13:138-44.

[31] Rehani MM. CT imaging in a large part of the world: what we know and what we can learn. Pediatr Radiol 2014;44(S3):511-4. https://doi.org/10.1007/s00247014-3045-6.

[32] Goske MJ, Strauss KJ, Coombs LP, Mandel KE, Towbin AJ, Larson DB, et al. Diagnostic reference ranges for pediatric abdominal CT. Radiology 2013;268(1): 208-18. https://doi.org/10.1148/radiol.13120730.

[33] Padole AM, Sagar P, Westra SJ, Lim R, Nimkin K, Kalra MK, et al. Development and validation of image quality scoring criteria (IQSC) for pediatric CT: a preliminary study. Insights Imaging 2019;10(1). https://doi.org/10.1186/s13244-019-0769-8.

[34] Kharita MH, AlNaemi H, Kini V, Al. E. Experience of integrating image quality with radiation dose in children undergoing CT examinations in Qatar. Eur Radiol 2020; In Press. 